\title{
An Investigation of Synchrony in Transport Networks
}

Rex K. Kincaid

William\&Mary, rrkinc@wm.edu

Natalia Alexamdrov

Michael J. Holroyd

Follow this and additional works at: https://scholarworks.wm.edu/aspubs

\section{Recommended Citation}

Kincaid, R. K., Alexandrov, N., \& Holroyd, M. J. (2009). An investigation of synchrony in transport networks. Complexity, 14(4), 34-43.

This Article is brought to you for free and open access by the Arts and Sciences at W\&M ScholarWorks. It has been accepted for inclusion in Arts \& Sciences Articles by an authorized administrator of W\&M ScholarWorks. For more information, please contact scholarworks@wm.edu. 


\title{
An Investigation of Synchrony in Transport Networks
}

\author{
REX K. KINCAID ${ }^{1}$, NATALIA ALEXANDROV ${ }^{2}$, AND MICHAEL J. HOLROYD ${ }^{3}$ \\ ${ }^{1}$ Department of Mathematics, The College of William and Mary, Williamsburg, Virginia; ${ }^{2}$ NASA Langley Research \\ Center, Hampton, Virginia; and ${ }^{3}$ Department of Computer Science, University of Virginia, Charlottesville, Virginia
}

Received February 27, 2007; accepted May 23, 2008

\begin{abstract}
The cumulative degree distributions of transport networks, such as air transportation networks and respiratory neuronal networks, follow power laws. The significance of power laws with respect to other network performance measures, such as throughput and synchronization, remains an open question. Evolving methods for the analysis and design of air transportation networks must be able to address network performance in the face of increasing demands and the need to contain and control local network disturbances, such as congestion. Toward this end, we investigate functional relationships that govern the performance of transport networks; for example, the links between the first nontrivial eigenvalue, $\lambda_{2}$, of a network's Laplacian matrix-a quantitative measure of network synchronizability - and other global network parameters. In particular, among networks with a fixed degree distribution and fixed network assortativity (a measure of a network's preference to attach nodes based on a similarity or difference), those with small $\lambda_{2}$ are shown to be poor synchronizers, to have much longer shortest paths and to have greater clustering in comparison to those with large $\lambda_{2}$. A simulation of a respiratory network adds data to our investigation. This study is a beginning step in developing metrics and design variables for the analysis and active design of air transport networks. (c) 2008 Wiley Periodicals, Inc. Complexity 14: 34-43, 2009
\end{abstract}

Key Words: networks; power laws; degree distribution; synchronization

\section{INTRODUCTION}

T he US and world-wide air transport networks are scalefree $^{1}$, i.e., their degree distributions follow power laws $[2,3]$. A large body of recent work has been published on scale-free networks including the popular books Linked

${ }^{1}$ We use the term "scale-free" here to denote networks whose degree distributions follow a power law. Because of a wide range of properties possessed by networks of similar degree distributions, there is an ongoing discussion of the meaning of "scale-free" [1].

Correspondence to: Rex K. Kincaid (e-mail:rrkinc@math.wm. edu) by Barabási [4] and Six Degrees by Watts [5]. A number of excellent review articles, including Newman [6], Strogatz [7], Albert and Barabási [8], and Dorogovtsev and Mendes [9], contain hundreds of references.

Arguably, many scale-free networks occurring in natural and technological realms have never been actively designed in the traditional sense: identify design variables, objectives, and constraints and follow a prescriptive algorithm to obtain a design that satisfies constraints and is "optimal" with respect to the given objectives. Rather, these networks have evolved in response to demands, in accordance with some natural or technological rules. Thus many of the investigations have been of an analytical nature, i.e., given a particular natural or technological network, its 
characteristics are studied. Our ultimate interest is to take a step from analysis to active design and our motivation comes from air transport systems. We emphasize that complex networks will likely never be completely amenable to traditional design methods, given the intrinsic lack of predictive modeling akin to that of physical artifacts (e.g., airplanes, automobiles). However, we conjecture that some measure of active design is still possible with the identification of appropriate design variables and metrics. Specifically, we are looking for an appropriate functional relation between global metrics (e.g., throughput, delays, capacity, synchronizability) and locally controllable structure (e.g., connectivity, degree).

Air transport networks are our ultimate domain of interest. Are scale-free networks desirable for air transport? Given a fixed degree distribution, how should the network links be configured to achieve optimal performance for relevant metrics? Can salient features of scale-free air transport networks critical to network performance be identified?

With the advent of deregulation of the US air transport system in 1978, airlines began to organize their operations with a hub-and-spoke approach. Two natural outcomes have been an increase in flight frequency and an increase in the variance of flight times. Recently, competitors to the hub-and-spoke model have garnered attention with the use of point-to-point flight schedules. With the delays experienced by travelers at hub airports these direct flights are an attractive alternative. How will these point-to-point airlines alter the air transport network structure? Can we provide any guidance to local air routing decisions with global air transport network performance measures in mind? To begin to address these questions, we examine the effects of a network metric for synchronization on transport route structure by holding the degree distribution and a scale-free/scale-rich metric constant. We also report on a simulation of a respiratory neuronal network used as an additional testbed for investigating the synchrony metrics. It is a first step in the investigation aimed at deriving the functional dependences among various local network properties and the aggregated metrics of interest to participants in the transportation system in an effort to eventually arrive at active transport network design and optimization algorithms.

\section{BACKGROUND}

In this section, we briefly review some of the network attributes salient to our investigation into the network functional relationships. Early network growth models were based on preferential attachment. A variety of authors have developed extensions and improvements to the early models. All of these mechanisms build networks sequentially, one node at a time. Barabasi's [4] original approach selected the end nodes for the edges associated with the new node based solely on the current network's degree distribution. Subsequent efforts have altered the end-node selection method to control other network features. For example, Wang et al. [10] developed a growth model in which assortativity is tunable, while Schank and Wagner [11] and Holme and Kim [12] grew networks with tunable clustering coefficients.

The network attribute under study here is synchronicity. One notion of synchronicity has to do with the network's tendency to synchronize over time, given a specific static structure of the network. In particular, we are not yet considering explicit traffic flows through the air transport network. Instead, we are investigating how the node (e.g., airport) connectivity may influence the traffic flow.

Of importance here is the tunability of a given network with respect to synchronicity. We realize tunability as network optimization. Before proceeding further, we provide a definition of network synchronization for a discrete complex system. Given a connected network, denote the state of a node $i$ at time $t$ by $x_{i}(t)$. How do the states of the nodes change over time? Clearly if nodes do not rely on any information generated by adjacent nodes then there is no opportunity of synchronization. Atay et al. $[13,14]$ assumed that all nodes are identical and conform to the following generic discrete time equation to determine their next state:

$$
x_{i}(t+1)=f\left(x_{i}(t)\right)+\kappa\left[\frac{1}{k_{i}} \sum_{j \mid(i, j) \in \text { Edges }} f\left(x_{j}(t)\right)-f\left(x_{i}(t)\right)\right] \text {, }
$$

where $\kappa$, known as the coupling constant, is a scalar describing the extent to which neighbors effect the state of a node; $f(\cdot)$ is any differentiable function mapping some finite interval to itself. The function $f(\cdot)$ describes the behavior of a node in the absence of any outside influence. We say that a network synchronizes for a given initial condition if for all $i, j$

$$
\lim _{t \rightarrow \infty}\left|x_{i}(t)-x_{j}(t)\right|=0 .
$$

Note that if $\kappa=0$, then the equation becomes $x_{i}(t+1)=$ $f\left(x_{i}(t)\right)$.

One attribute that correlates with the network's capacity to synchronize is the first nontrivial eigenvalue, $\lambda_{2}$, of the Laplacian matrix associated with the network structure (more about $\lambda_{2}$ in the next section). Here $\lambda_{2}$ acts as a measure of the range of $\kappa$ over which the network will synchronize.

Clearly, air transport networks will not meet some of the assumptions. The nodes (airports) are not all identical and we are more interested in the transient (say, 24-h period) behavior than in what happens as $t \rightarrow \infty$. Nonetheless, in the next section we will see that $\lambda_{2}$ will provide useful information with regard to network structure and synchrony. 
For some complex systems, synchronization is an essential feature. For example, in mammals a small group of neurons (roughly 200) is responsible for generating a regular rhythmic output to motor cells that initiate a breath. (We explore this example further in Section 4.) Without synchronization of the neuronal output, breathing would be ragged or not occur at all. However, in the defined sense, synchronization is an undesirable attribute for air transport networks. Think of the airports as neurons in our mammalian respiratory example. Inhaling means all planes land at all airports simultaneously. Exhaling means they depart together. The result is congestion. Thus, for the given definition, one would like an air transport network design to minimize synchronization.

Another network metric, developed in [1], serves to classify the ways in which networks with a given degree distribution may be constructed. The metric is $s(G)$, where $G$ stands for "graph" (networks and graphs are interchangeable). To determine $s(G)$, compute the product of the degrees of the end nodes for each edge or link, sum them up for all edges, and divide by $s_{\max }$, where $s_{\max }$ is the maximum value of the sum taken over all possible connected graphs for a fixed degree distribution. That is,

$$
s(G)=\sum_{(i, j) \in \text { Edges }}\left(d_{i} \times d_{j}\right) / s_{\max },
$$

where $d_{i}$ is the degree of node $i$. The value of $s_{\max }$ provides a way to scale the sum of the product of the degrees for each edge. In [1], for a fixed degree distribution, graph realizations with large values of $s(G)$ are termed scale-free and graph realizations with small values of $s(G)$ are termed scalerich. Consequently, scale-free graphs or networks are those in which high-degree nodes are more likely to be adjacent to other high-degree nodes, whereas scale-rich graphs are those for which high-degree nodes are more likely to be adjacent to low-degree nodes. As shown in [1], $s(G)$ and assortativity are equivalent but are scaled in different ways. Typically, the scaling for the assortativity measure leads to a tighter range of values for a given degree distribution. For further information about $s(G)$ and how it is used to distinguish between networks for the Internet at the router level, the interested reader is referred to [1] and [15].

The problems faced by designers of air transport networks share some aspects with the design of an Internet router network. Many authors have contributed to investigations of how a router network is constructed. Two references in this field, [1] and [15], contain ideas central to our consideration of the design of air transport networks. At one level of resolution, Table 1 points out the analogies between these two network design problems. With regard to bandwidth, the Internet router designer must weigh the trade-offs between many low bandwidth connections and fewer high bandwidth connections. These trade-offs are akin to choosing between
TABLE I

Analogy Between Internet Router and Air Transport Networks

\begin{tabular}{lll}
\hline & Internet & Air Transport \\
\hline Product & Packets & Planes (loaded) \\
Constraint & Bandwidth & Airport capacity \\
Competitors & ISPs & Airlines \\
Links & Hardwired & FAA/Airlines \\
Distributors & Routers & Airports
\end{tabular}

a few hub airports in a hub-and-spoke system and choosing lower frequency airports that might arise in a direct route system. Of course, there are many differences as well. The variation in the size of the packets for the Internet is not nearly as great as the number of passengers on planes of different sizes. In addition, although the FAA ${ }^{2}$ clearly defines the routes allowed between airports, the links are as not hard-wired as they are in the Internet model. Still there is much to be learned from the research efforts on the design of effective Internet router networks.

\section{NETWORK EXPERIMENTS WITH $\lambda_{2}$}

In this section, the interplay between $\lambda_{2}$, a gross measure of network synchrony, and $s(G)$, a network measure similar to assortativity is examined. Networks of two typespreferential attachment and geometric-are the testbeds. Each network has 100 nodes and is a simple undirected network (no self-loops, no multiple edges). An adjacency matrix, $A$, is constructed. $G(V, E)$ denotes a graph (network) with vertex (node) set $V$ and edge (link) set $E$. For each network type, the degree distribution and $s(G)$ values are fixed. Finally, a tabu search heuristic rewires a given network so as to minimize (or maximize) the value of $\lambda_{2}$.

Preferential attachment graphs are generated following the approach given in [4]. The network is grown by adding nodes and edges. For each node added, $m$ edges are added preferentially, based on the current degree distribution. Geometric graphs are generated by randomly selecting 100 points $(r, \theta)$ with values of $r \in[0,1]$ and values of $\theta \in(0,360]$. Edges exist between pairs of points if the Euclidean distance is less than a specified threshold (in our experiments thresholds between 0.17 and 0.25 were used). If the resulting graph is connected, it is kept; otherwise it is rejected and the process begins again. A variety of network performance measures are available. These include network diameter, average degree, assortativity, clustering coefficient, synchrony, and $s(G)$.

\footnotetext{
${ }^{2}$ Federal Aviation Administration.
} 


\section{FIGURE 1}

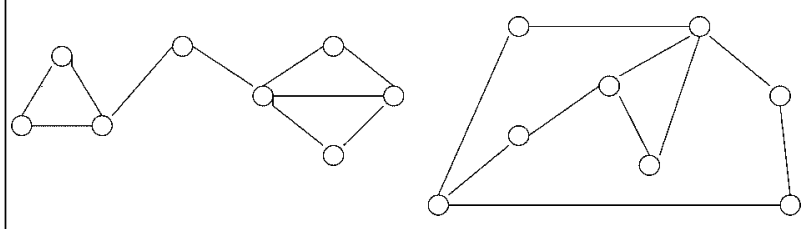

Geometric graph with $\lambda_{2}=0.238$ and $\lambda_{2}=0.925$.

Our measure of synchrony relies on computing an eigenvalue of the Laplacian matrix associated with a given network structure. For our networks or graphs, the Laplacian matrix is a symmetric matrix $L=D-A$, where $D$ is a diagonal matrix with the degree of each node located along the main diagonal. $A$ is the adjacency matrix for the graph. The second eigenvalue of $L$ measures algebraic connectivity [16]. Intuitively, graphs with small $\lambda_{2}$ are easier to "pull apart". In particular, if $\lambda_{2}=0$, then the graph is disconnected. Many authors $[13,17,18]$ have convincingly used $\lambda_{2}$ as a global measure of how likely a graph is to synchronize. That is, given an arbitrary flow of entities, the graph is less likely to synchronize if $\lambda_{2}$ is small. The two graphs in Figure 1 have identical degree distributions, but the graph on the left is more weakly connected (e.g., the removal a single edge can disconnect the graph). The identification of structural differences between large graphs with varying values of $\lambda_{2}$ is studied in the following set of numerical experiments.

In the remainder of the section, we describe numerical experiments in optimizing two types of networks for $\lambda_{2}$ : geometric graphs with 100 nodes and preferential attachment graphs with $m=2$.

The graphs plotted in Figures 2 and 3 were constructed by first generating a random instance of the particular graph class-geometric in Figure 2 or preferential attachment in Figure 3. Next, a simple tabu search [19] heuristic was called to minimize or maximize $\lambda_{2}$ while keeping the degree distribution and $s(G)$ fixed. Allowable moves (re-wirings) are pair-wise edge interchanges that preserve the degree distribution and $s(G)$. Briefly, the tabu search checks to see if the move is acceptable, that is, if the move is improving and not tabu, or improving and tabu but leads to the best observed value of $\lambda_{2}$ (aspiration criterion). Note that these moves are precisely the moves allowed in a random rewiring scheme without checking for the preservation of $s(G)$. The interested reader is referred to Glover and Laguna [19] for further information on tabu search.

Figures 2-4 display networks with respect to the reciprocal of the eccentricity of each node $u$. The eccentricity of $u$ is its maximum (shortest path) distance. The graphs are generated by socnetv ${ }^{3}$. The goal of the plots is to uncover any qualitative differences between the graphs with small and large values of the second eigenvalue of the Laplacian. ${ }^{4}$ For each pair of plots in a figure, the number of nodes (100), the degree distribution, and the value of $s(G)$ is fixed.

Nodes with equal eccentricity values are plotted on the same (dashed line) circles. The circles with larger radii have larger eccentricity. Consequently, nodes near the center have shorter longest paths. The paired plots exhibit large qualitative differences in the eccentricity patterns. The same pairs of plots for other available measures in socnetv were also constructed. Although small variations were seen, none of the other paired plots exhibited significant differences.

Qualitatively, when $\lambda_{2}$ is small, the patterns are less organized, the eccentricity plots in Figures 2(a) and 3(a) are more dispersed and consist of many rings of constant eccentricity. The eccentricity plots with larger $\lambda_{2}$ are more organized, with few rings of constant eccentricity. Specifically, the plots with small $\lambda_{2}$ have 11 [Figure 2(a)] and 10 [Figure 3(a)] rings. For larger $\lambda_{2}$, there are five [Figure 2(b)] and four [Figure 3(b)] rings, respectively. The ranges of eccentricity values for the small $\lambda_{2}$ plots are dominated by the ranges for the large $\lambda_{2}$ plots. For example, the range of eccentricity values for the geometric graph with small $\lambda_{2}$ [Figure $\left.2(a)\right]$ is $[26,42]$ and for the geometric graph with large $\lambda_{2}$ [Figure 2(b)] it is [4, 8]. Thus, the patterns of eccentricities in Figures 2(b) and 3(b) are nonoverlapping and dominate those given in Figures 2(a) and 3(a).

The diameter of the graph in Figure 2(a) is 42 , whereas the graph diameter in Figure 2(b) is 8 . The diameters are 19 and 6 for graphs in Figure 3. Notice that this is also true for the simple graphs in Figure 1. The graph on the left has a larger diameter than the one on the right. For graphs with a fixed degree distribution and a fixed value of $s(G)$, this result appears to hold in general. We know of no theorem that proves this result but numerous computational tests support this claim so far. Moreover, the inverse relationship between $\lambda_{2}$ and the eccentricity does not hold if $s(G)$ is allowed to vary. Figure $4(\mathrm{a}, \mathrm{b})$ provide an example. The value of $\lambda_{2}=0.935$ in Figure 4(a) is larger than $\lambda_{2}=0.440$ in Figure 4(b). Yet the range of eccentricity values for Figure $4(a),[8,16]$, is larger and does not overlap with the range for Figure $4(\mathrm{~b})$ of $[3,6]$.

In Figures 5 and 6, we investigate the relation between $s(G)$ and the clustering coefficient, $c(G)$ [6], of a network or graph. These figures display results for 5000 geometric graphs. In Figure 5, the 5000 geometric graphs are generated randomly.

\footnotetext{
${ }^{3}$ The source code and documentation can be found at http: //socnetv. sourceforge. net/.

${ }^{4}$ We leave it to the reader to become acquainted the variety of measures and display features in socnetv. For the purposes of this exposition, we are interested only in the qualitative differences between the plots.
} 


\section{FIGURE 2}

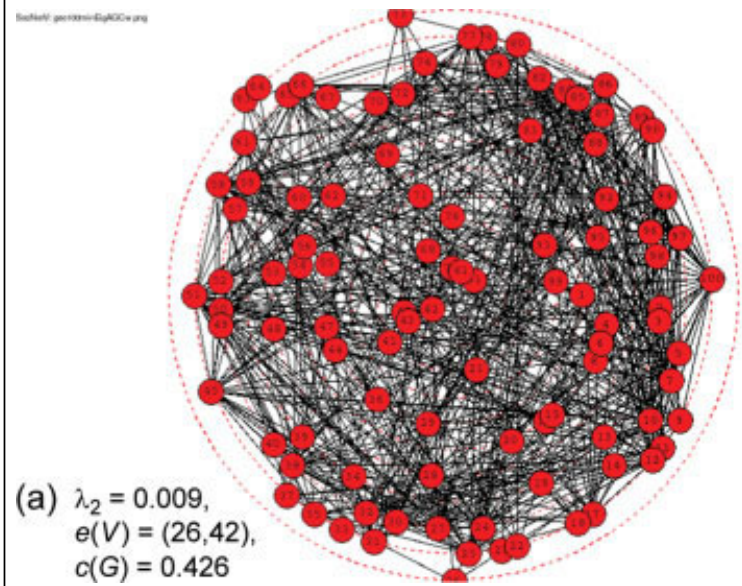
(b) $\lambda_{2}=0.314$,
$e(V)=(4,8)$,
$c(G)=0.297$

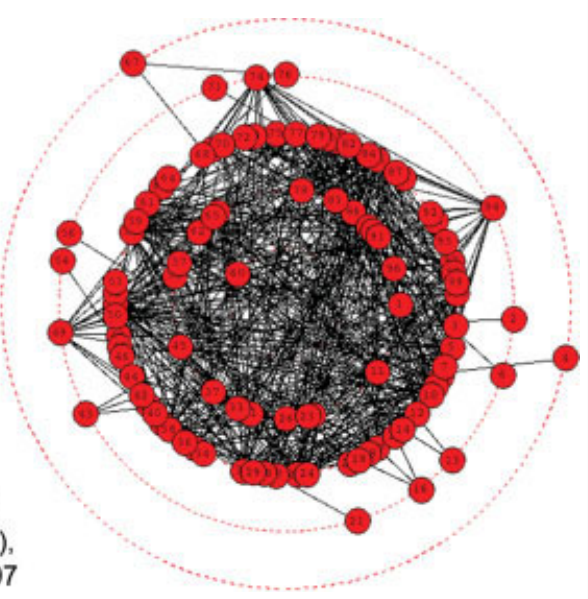

Geometric graphs: 100 nodes, $s(G)=0.971$, fixed degree distribution. [Color figure can be viewed in the online issue, which is available at www.interscience.wiley.com.]

Edges connect nodes if the Euclidean distance between these nodes is less than 0.23 . If the resulting graph is connected, it is kept; otherwise it is rejected and the process begins again. Figure $5(\mathrm{~b})$ displays the values of $s(G)$ versus $c(G)$. There is no apparent correlation. Figure 6(a) records the values of $s(G)$ when a given 100-node geometric graph is randomly rewired 5000 times. Figure 6(b) illustrates the inverse relationship between $s(G)$ and $c(G)$ when the first 364 re-wirings are excluded.
In addition to the inverse relationship between $\lambda_{2}$ and the eccentricity, the clustering coefficient varies inversely with $\lambda_{2}$ in Figures 2 and 3. Notice that when $s(G)$ is not held constant, as in Figure 4, this relationship does not hold. A similar trend between $s(G)$ and $c(G)$ is observed in Figure 6. Figure 6(a) displays the value of $s(G)$ versus 5000 random re-wirings of a given geometric graph. We note that the same moves as those used in our tabu search to optimize $\lambda_{2}$ are used for the random re-wirings. Figure 6(b) plots $s(G)$ versus $c(G)$ for the

FIGURE 3

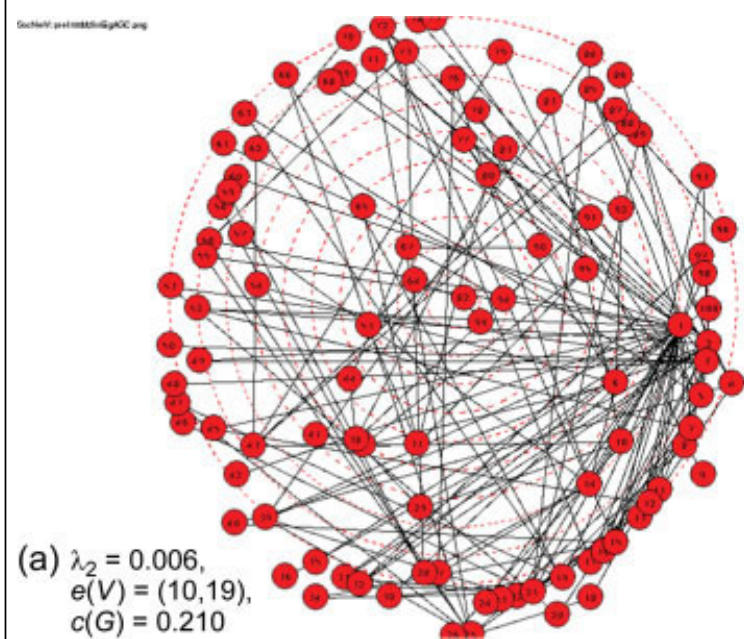
(b) $\lambda_{2}=0.365$, $e(V)=(3,6)$ $c(G)=0.101$

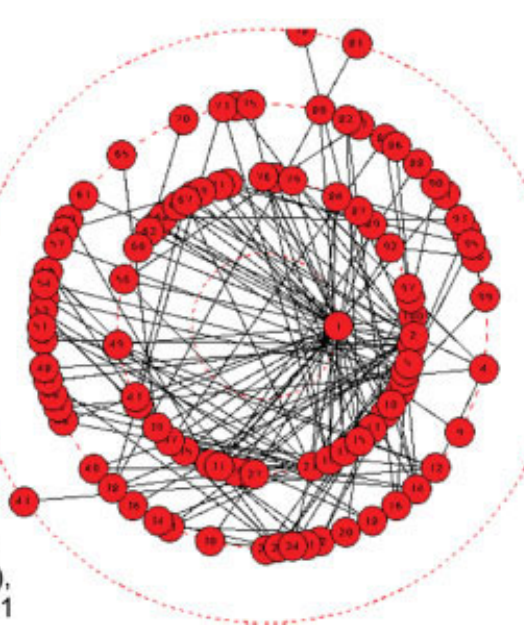

Preferential attachment: 100 nodes, $s(G)=0.716$, fixed degree distribution. [Color figure can be viewed in the online issue, which is available at www.interscience.wiley.com.] 


\section{FIGURE 4}
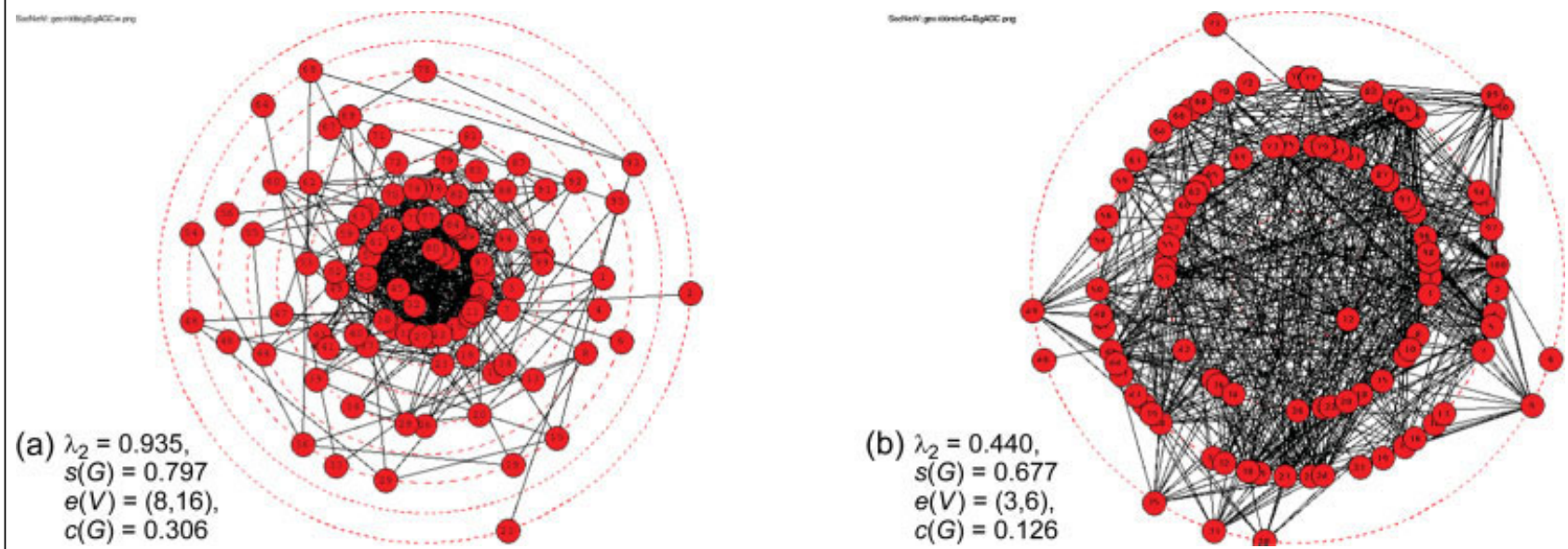

Geometric graphs: 100 nodes, fixed degree distribution, varying $s(G)$. [Color figure can be viewed in the online issue, which is available at www.interscience.wiley.com.]

last 4634 random re-wirings and $s(G)$ varies inversely with $c(G)$. Notice that in Figure 6(a), the first 600 or so random rewirings decrease $s(G)$ almost monotonically before settling into an oscillating pattern of increases and decreases in the range of $(0.79,0.83)$. This is not the case, however, when geometric graphs are generated at random (no rewiring), as in Figure 5. Here no correlation is exhibited between $c(G)$ and $s(G)$.

As we have noted earlier, Figure 4(a,b) illustrates that $s(G)$, or some yet unknown network metric, appears to exert significant influence on the eccentricity pattern. In Figure 2(b), $\lambda_{2}=0.314, s(G)=0.971$, and the eccentricity range is $[4,8]$.
This compares favorably with the results in Figure 4(b), where $\lambda_{2}=0.440$ is larger and the eccentricity range of $[3,6]$ is smaller with a smaller minimum node eccentricity. However, the comparisons with Figure 4(a) are not consistent. The value for $\lambda_{2}$ in Figure 4(a) is larger but, unexpectedly, the max and min values for the eccentricity range are much larger than those in Figure 2(b). In addition, the previously observed pattern of $c(G)$ varying inversely with $\lambda_{2}$ no longer holds. For example, $\lambda_{2}$ decreases from 0.935 in Figure 4 (a) to 0.440 in Figure 4(b). Similar decreases observed in Figures 2 and 3 led to a doubling of $c(G)$. But here $c(G)$ decreases by more than a half. It is unclear if the role of $s(G)$ explains the lack of

\section{FIGURE 5}
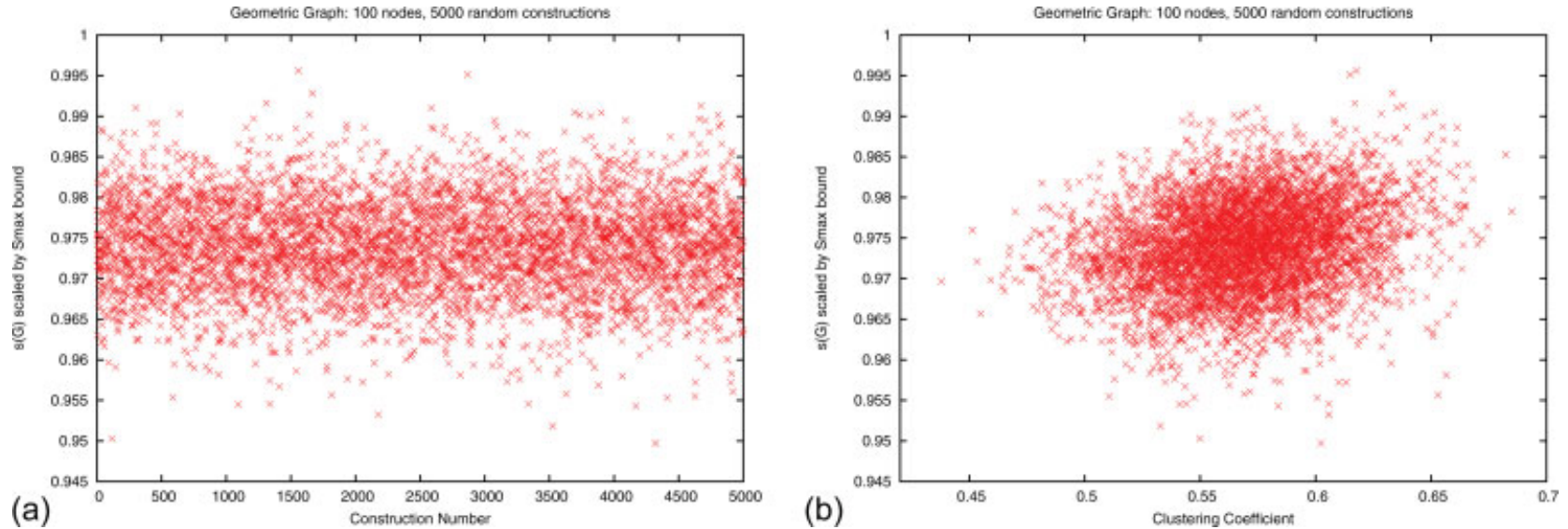

5000 random geometric graphs. [Color figure can be viewed in the online issue, which is available at www.interscience.wiley.com.] 


\section{FIGURE 6}
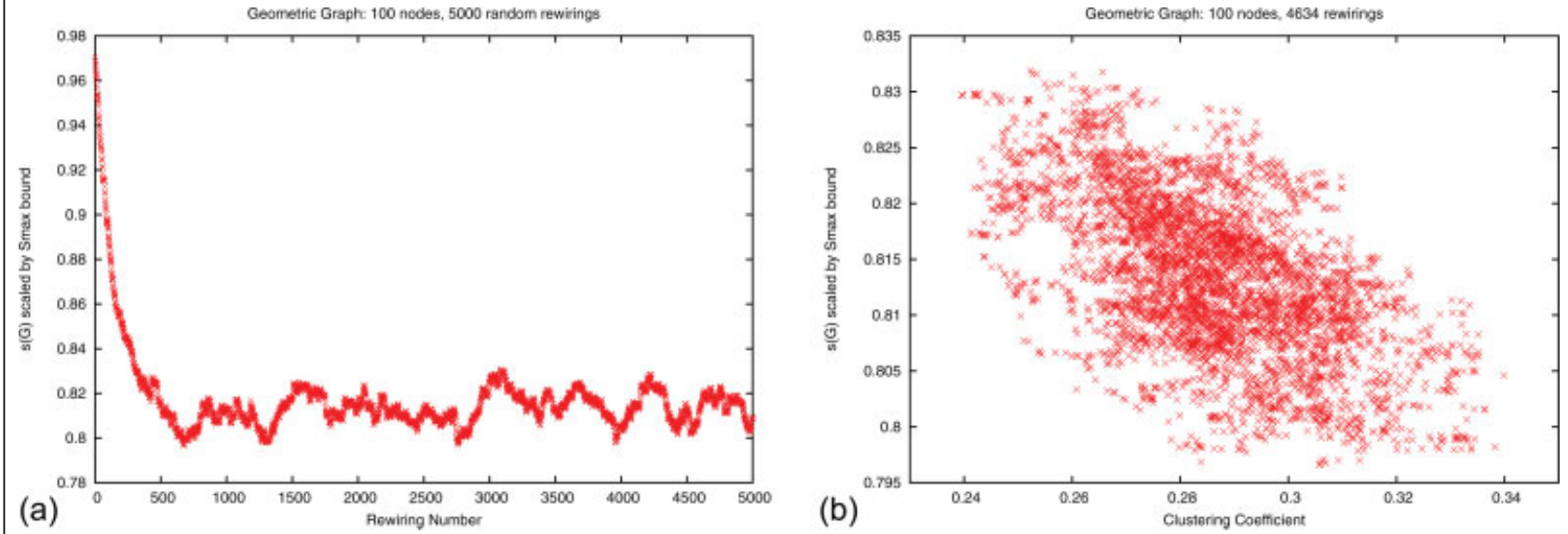

5000 re-wirings of geometric graphs. [Color figure can be viewed in the online issue, which is available at www.interscience.wiley.com.]

consistency. Figure 5 provides one possible explanation. Here geometric graphs are generated at random (no rewiring) and there is no correlation between $s(G)$ and $c(G)$. In Figure 6, an inverse correlation exists but here the graphs are constructed by successively rewiring a single geometric graph at random.

\section{RESPIRATORY NETWORK SIMULATION}

In the previous section, we have seen that when the degree distribution and $s(G)$ are fixed, there is a predictable difference in the shortest path distribution (eccentricity measures) and the clustering coefficients. Ideally, the next step would be to simulate the performance of networks presented in the previous section and investigate their performance as air transport network systems. We are currently proceeding in this direction using a model previously developed to simulate the complete US air transport system. For now, we provide simulation results for a different system-a respiratory neural network - for which a simpler simulation model was readily available. In this model, synchronization (rhythmic breathing) is desired.

\section{FIGURE 7}
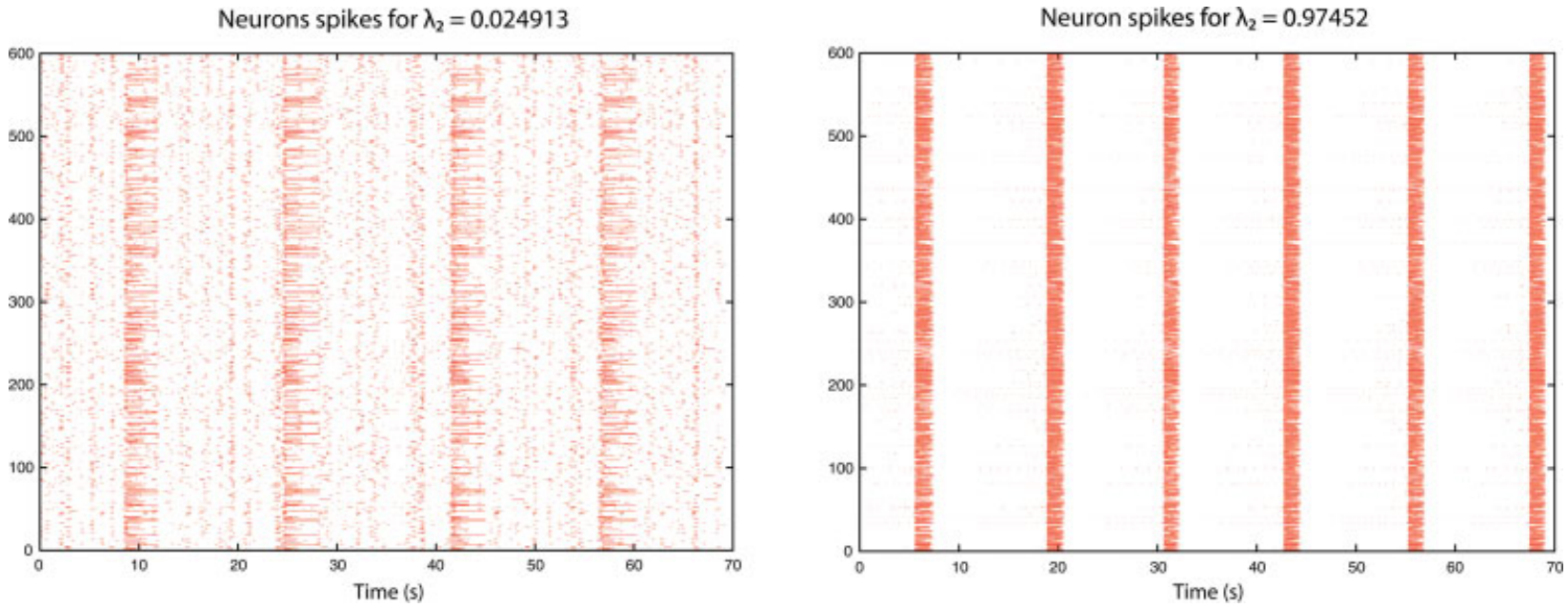

Raster plots of neuron output for two networks with disparate $\lambda_{2}$ values. A point at $(x, y)$ indicates neuron $x$ is spiking at time $y$. The higher $\lambda_{2}$ network displays much stronger synchronization among all nodes as predicted, as well as a quicker breath frequency. [Color figure can be viewed in the online issue, which is available at www.interscience.wiley.com.] 

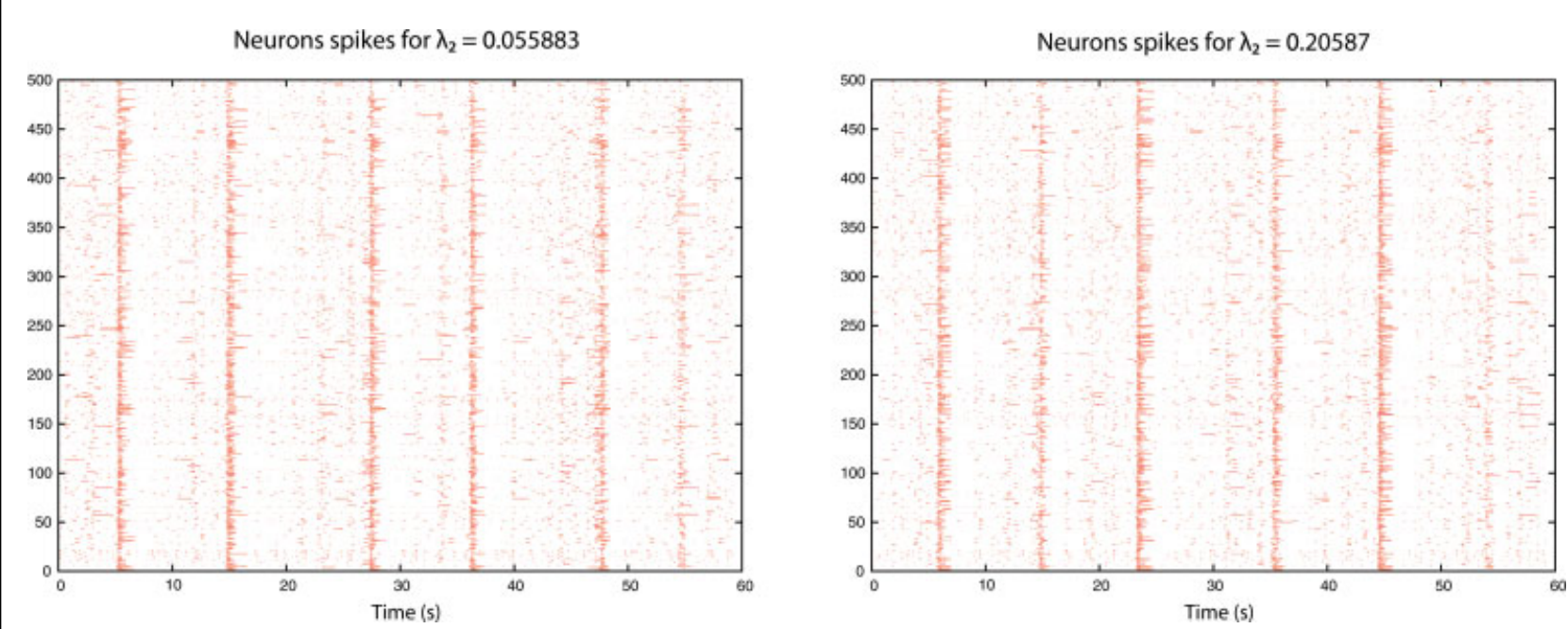

Indistinguishable raster plots of simulated neuron output for two sample networks with differing $\lambda_{2}$ values. [Color figure can be viewed in the online issue, which is available at www.interscience.wiley.com.]

Although simple synchronization is undesirable for air transport networks, there are systems for which it is an essential feature. In mammals, a small group of neurons is responsible for generating a regular rhythmic output to motor cells that initiate a breath. The network structure of these neurons allows them to synchronize without any external influence and produce regular bursts that lead to breaths. In [20], two geometric networks, one with a value of $\lambda_{2}=0.025$ and a second with a value of $\lambda_{2}=0.974$ were tested in a simulation model [21] of this neuronal network. The rhythmic output from the the network with $\lambda_{2}=0.025$ was ragged with fuzzy bursts, whereas outputs from the network with $\lambda_{2}=0.974$ was sharp with clear, regular bursts (Figure 7).

In mammals, a small group of neurons in the brain stem, called the pre-Bötzinger complex, is responsible for generating a regular rhythmic output to motor cells that initiate a breath. Disconnected, these neurons are unable to provide sufficient output to activate the motor neurons, but their interconnected network structure allows them to synchronize without any external influence and produce regular bursts. Using a detailed simulation by Hayes [21], we were able to experiment with how different network topologies control the effectiveness of the pre-Bötzinger complex. We began by testing two geometric graphs with extreme values of $\lambda_{2}$. The results of the two simulations, depicted in Figure 7, provide compelling evidence for the utility of $\lambda_{2}$ as a predictor of synchronization. It is easy to see that the network with higher $\lambda_{2}$ synchronizes more strongly than the other network. The second set of simulations investigated two preferential attachment networks. The raster plots in Figure 8 are nearly indistinguishable. The results of the simulation are further analyzed via an autocorrelation analysis (Figure 8). Analysis (as in [21]) uncovers better synchronization in the network with the higher value of $\lambda_{2}$. The results in Figure 9 confirm that, although the difference is undetectable at a first glance (Figure 8), higher $\lambda_{2}$ results in a better synchronization. Autocorrelation indicates the largest difference during the refractory (non-spiking) period: the two graphs exhibit similar behaviors during spikes, but not between spikes. These experiments provide further evidence that $\lambda_{2}$ can be used to identify graphs (networks) that are not likely to synchronize.

\section{FIGURE 9}

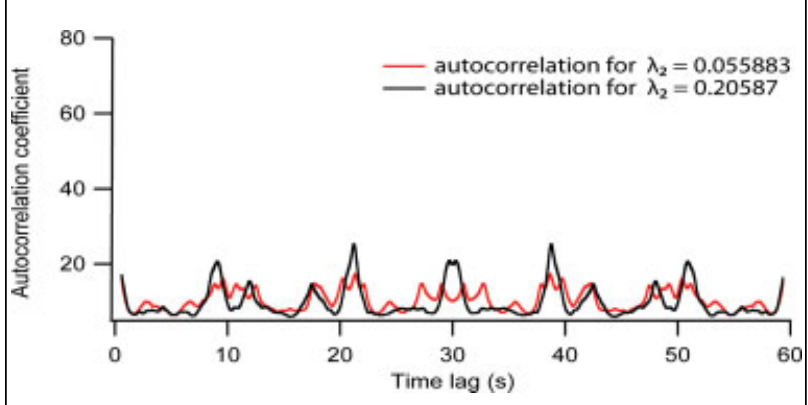

An autocorrelation plot of pre-Bötzinger complex synchronization on two networks with the same degree distribution, but with differing $\lambda_{2}$ values. The autocorrelation analysis shows that the higher $\lambda_{2}$ network displays better synchronization. [Color figure can be viewed in the online issue, which is available at www.interscience.wiley.com.] 


\section{CONCLUSIONS AND DISCUSSION}

Given the present state of air transportation networks, there is some urgency in developing active and rigorous design methodologies. Our goal is to develop a systematic way to design for some salient aspects of air transport networks. In particular, network structure, both static (node location) and dynamic (air route scheduling) has a direct effect on the functioning of the traffic in the network; we are now concerned with the effect of the static and dynamic network structure on the performance.

How should the design process proceed? Design involves being able to manipulate variables so as to optimize objectives subject to constraints. As a step in this direction, we have demonstrated that for a fixed degree distribution and fixed $s(G)$ value (and, consequently, a fixed assortativity), optimizing for $\lambda_{2}$ yields networks with distinct eccentricity patterns. We have demonstrated the ability to construct networks with locally optimal $\lambda_{2}$ and observed a correlation with global network attributes, such as clustering, eccentricity, and synchronizability. These results are further supported by a simulation analysis of another transport system - a respiratory neuronal network. This simulation supports our conjecture that large differences in $\lambda_{2}$ result in observable differences in the burst activity: good synchronization for large $\lambda_{2}$ and poor synchronization for small $\lambda_{2}$. It remains to simulate air transport networks with small and large values of $\lambda_{2}$ (more than likely with identical degree distributions and $s(G)$ values). In addition to validating the static results for networks (as in Figures 2 and 3), the simulation will also measure quantities of interest to the FAA that are currently not amenable to rigorous optimization. For example, the simulation will measure congestion effects in air traffic sectors. Finally, one of our major tasks is to derive maps between the network metrics we can control and airspace simulations and, ultimately, FAA metrics. We conjecture that deriving the maps will enable active design for a number of objectives and constraints.

A few words about practical matters are in order. We realize that the traditional research in transportation tends to be of a more immediately applied nature. The line of inquiry we are pursuing is very much in its infancy and we cannot even refer (to the best of our knowledge) to similar publications in transportation research. Our only references to similar network investigations are thus far in the realm of the Internet $[1,15]$. However, we firmly believe that the ongoing difficulties in implementing profound changes in the present air transportation system (due, in general, to its immense complexity) can be, in particular, traced to the lack of predictive modeling. To arrive at predictive modeling-or to understand the limitations of possible modeling-we must start with an investigation into functional relationships that, at first, appear theoretical and somewhat removed from the practicalities of the system. Fortunately, there is a growing recognition of the need for such fundamental inquiries into the nature of complex networks. For instance, a recent NASA Research Announcement explicitly targeted basic research into modeling and active design of transport networks [22]. Thus, we hope that, should an initial emphasis on theory lead to a better understanding of network behavior and to quantitative analysis and design algorithms, we would meet a receptive audience in the transportation community.

\section{ACKNOWLEDGMENTS}

The authors kindly thank the anonymous referee for suggestions that improved the final version of this work.

\section{REFERENCES}

1. Li, L.; Alderson, D.; Doyle, J.C.; Willinger, W. Towards a theory of scale-free graphs: Definition, properties, and implications. Internet Math 2006, 2 , 431-523.

2. Guimera, R.; Mossa, S.; Turtschi, A.; Amaral, L.A.N. Structure and efficiency of the world-wide airport network, Preprint 2003, 0312535. Available at http://arxiv.org/abs/cond-mat/.

3. Guimera, R.; Amaral, L.A.N. Modeling the world-wide airport network. Eur Phys J B 2004, 38, 381-385.

4. Barabási, A.-L. Linked: The New Science of Networks; Perseus: Cambridge, MA, 2002.

5. Watts, D.J. Six Degrees: The Science of a Connected Age; Norton: New York, 2003.

6. Newman, M.E.J. The structure and function of complex networks. SIAM Rev 2003, 45, 167-256.

7. Strogatz, S.H. Exploring complex networks. Nature 2001, 410, 268-276.

8. Albert, R.; Barabási, A.-L. Statistical mechanics of complex networks. Rev Mod Physics 2002, 74, 42-47.

9. Dorogovtsev, S.N.; Mendes, J.F.F. Evolution of networks. Adv Phys 2002, 51, 1079-1187.

10. Wang, W.-X.; Hu, B.; Wang, B.-H.; Yan, G. Available at: arXiv:cond-mat/0505419 v1, May 2005.

11. Schank, T.; Wagner, D. Approximating clustering coefficient and transitivity. J Graph Algorithms Appl 2005, 9, 265-275.

12. Holme, P.; Kim, B.J. Growing scale-free networks with tunable clustering. Phys Rev E 2002, 65, 026107.

13. Atay, F.M.; Jost, J. Delays, connection topology, and synchronization of coupled chaotic maps. Phys Rev Lett 2004, 92, 144101(4).

14. Atay, F.M.; Biyikoglu, T.; Jost, J. Synchronization of networks with prescribed degree distributions. Available at: arXiv:nlin.A0/0407024 v2, May 2005.

15. Li, L.; Alderson, D.; Doyle, J.C.; Willinger, W. A first-principles approach to understanding the internet's router-level topology, Proc ACM SIGCOMM, Comp Comm Rev 2004, 34, 3-14.

16. Fiedler, M. Algebraic connectivity of graphs. Czech Math J 1973, 23, 298-305. 
17. Barahona, M.; Pecora, L.M. Synchronization in small-world networks. Phys Rev Lett 2002, 89, 054101-1-054101-4.

18. Wu, C.W. Perturbation of coupling matrices and its effect on the synchronizability in arrays of coupled chaotic systems, Preprint 2006, 0307052. Available at: http://arXiv/pdf/nlin.CD/.

19. Glover, F.; Laguna, M. Tabu Search. Kluwer Academic Publishers: Boston, MA, 1997.

20. Holroyd, M. Synchronizability and connectivity of discrete complex systems, Undergraduate Honors Thesis, Department of Mathematics, College of William and Mary, Williamsburg, VA, 2006.

21. Negro, C.D.; Hayes, J.; Morgadi-Valle, C.; Mackay, D.D.; Pace, R.W.; Crowder, E.A.; Feldman, J.L. Sodium and calcium current-mediated pacemaker neurons and respiratory rhythm generation. J Neurosci 2005, 25, 446-453.

22. NASA Research Announcement, Harry N. Swenson, Project Prinicpal Investigator, Aeronautics Research Mission Directorate, Solicitation: NNH07ZEA001N, 2007. 